\title{
Quality prediction modeling for sintered ores based on mechanism models of sintering and extreme learning machine based error compensation
}

\author{
Wu Tiebin, Liu Yunlian, Li Xinjun, Yu Yi, and Zhang Bin \\ Hunan University of Humanities, Science and Technology, Loudi, Hunan 41700, China
}

\begin{abstract}
Aiming at the difficulty in quality prediction of sintered ores, a hybrid prediction model is established based on mechanism models of sintering and time-weighted error compensation on the basis of the extreme learning machine (ELM). At first, mechanism models of drum index, total iron, and alkalinity are constructed according to the chemical reaction mechanism and conservation of matter in the sintering process. As the process is simplified in the mechanism models, these models are not able to describe high nonlinearity. Therefore, errors are inevitable. For this reason, the time-weighted ELM based error compensation model is established. Simulation results verify that the hybrid model has a high accuracy and can meet the requirement for industrial applications.
\end{abstract}

\section{Introduction}

Iron ores are raw materials in steel industry. After large sale mining for a long term, the reserves of natural rich ores have declined rapidly. In such context, poor ores have to be used to cope with the fact that the iron ore resources are increasingly scarce. However, poor ores cannot be directly put in blast furnaces, which otherwise would cause abnormal production under severe circumstances. Therefore, poor ores need to be agglomerated before being put into blast furnaces. In China, the agglomeration of more than $90 \%$ of poor ores is realized using sintering process 错误!未找到引用 源。. The sintering of iron ores refers to a process of roasting several types of iron ore powders, flux, and coke at high temperature to sinter and agglomerate the iron ore powders on incomplete melting conditions. The quality of sintered ores directly influences the yield and efficiency of blast furnaces, so accurately predicting technical indices including iron grade, alkalinity, and drum index of sintered ores is the precondition to effectively improve the quality of sintered ores. However, as sintering is a multi-variable, highly-nonlinear, and strongly-coupled complex process with large delay and coexistence of multiple reactions ${ }^{2}$, accurately predicting the quality of sintered ores is always a challenge. According to the recondensation mechanism of water and the formation mechanism of over-wet zones, F. Patission established the mathematical model for the water migration in the sintering of iron ores. The model describes the relationship between the drum index and the liquid phases ${ }^{3}$. In previous research ${ }^{[4]}$, the mechanism model for the sintering process was built, and then the programming-genetic algorithm was applied

\footnotetext{
* Corresponding author: :Liu Yunlian, E-mail:liuyunlian85@163.com
}

to identify the parameters therein. Finally, simulation proves that the model is effective. Literature [5] concretely elaborated the construction of sub-mechanism models including the combustion of coke, drying and condensation of fritted hearth, oxidation of iron ores, melting and solidification of minerals, and heat and mass transfer of fritted hearth. In addition, the models were verified to be reasonable based on the experimental data of sintering pots. As the pure mechanism modeling has to simplify the sintering process and make assumptions, it is inevitable that the mechanism models show certain errors with the actual condition. For this reason, mechanism models can hardly describe the coupling and high nonlinearity of the process. Aiming at this, the errors in the prediction of the mechanism models are adopted as the modeling objective to build the error compensation model based on time-weighted extreme learning machine (ELM). Then the errors predicted using the data models are compensated to the output of the mechanism models. The hybrid modeling ensures that the prediction model has strong generalization ability and accuracy.

\section{Description of sintering process}

In the sintering process, different iron ores and coke are blended at first and then mixed with some screened return fines, followed by burden distribution. Afterwards, the mixture is sintered at appropriate ignition temperature and moment, and the sintering duration is determined by the movement speed of pallets. The sintered ores are crushed, screened, and cooled to produce the finished products of sintered ores. The 
screened fine ores are mixed with the raw materials and sintered again.

There are many factors influencing the quality parameters (such as iron grade, alkalinity, and drum index) of sintered ores, including mixing ratio of raw materials, executing ratio of powdered coke, ignition temperature, bed depth, and movement speed of pallets. The mixing ratio of raw materials mainly involves $\mathrm{FeO}$ content, TFe (total iron) content, water content, and ratio of limestone.

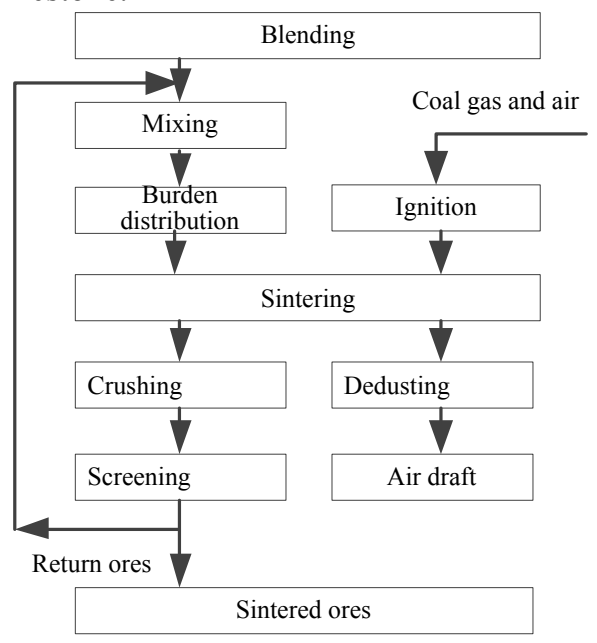

Figure 1 Flowchart of sintering process

\section{Mechanism modeling of the sintering process}

The quality of sintered ores greatly influences the yield and efficiency of blast furnaces, so accurately predicting technical indices of sintered ores including iron grade, alkalinity, and drum index is the premise of effectively improving the quality of sintered ores.

\subsection{Mechanism modeling of drum index of sintered ores}

Factors most greatly influencing the drum index include coke content, mass ratio of $\mathrm{CaO}$ to $\mathrm{SiO}_{2}$, and percentage content of $\mathrm{FeO}$ in the mixture as well as movement speed of pallets. On this basis, the following formula is established:

$$
Y_{d r u m}=\beta_{1}+\beta_{2} * x_{c}+\beta_{3} * x_{C a S i}+\beta_{4} * x_{F e O}+\beta_{5} * x_{v}
$$

Where $Y_{\text {drum }}$ represents the drum index; $x_{c}, x_{\text {CaSi }}$, and $x_{\mathrm{FeO}}$ denote the percentage content of coke $\left(2 \%<x_{c}<7 \%\right)$, mass ratio of $\mathrm{CaO}$ to $\mathrm{SiO}_{2}$ $\left(1.5<x_{\text {CaSi }}<3\right)$, and the percentage content of $\mathrm{FeO}$ $\left(2 \%<x_{F e O}<7 \%\right.$ ), in the raw materials, respectively; and $x_{v}$ refers to the movement speed of pallets.

\subsection{Modeling of TFe(Total Ferrum) content in sintered ores}

According to the conservation principle of matter, suppose that $100 \mathrm{~kg}$ of mixture is prepared and the percentage content of each type of iron concentrates is $x_{i}(i=1,2, \mathrm{~L}, N)$, then the mass fraction of total iron in the mixture is expressed as Formula (2).

$T F e_{\text {mix }}=\frac{\sum_{i=1}^{N}\left(100 * x_{i} * T F e_{i}\right)}{100}$

Where $T F e_{N}$ represents the iron grade of the mixture.

Production practice shows that the total iron in the mixture is basically consistent with that in the sintered ores. While, due to the consumption of all types of refined iron-mineral powders and powdered coke, the iron grade of sintered ores is higher than that of the mixture. The mechanism model for the total iron grade of the sintered ores is shown as Formula (3).

$$
Y_{T F e}=\frac{T F e_{\text {mix }} \times 100}{100-\sum_{i=1}^{n}\left(100 \times x_{i} \times d_{i}\right)}\left(\lambda_{0}+\lambda_{1} * x_{c}+\lambda_{2} * x_{c}^{2}\right)
$$

Where $d_{i}$ represents the burning loss rate of each concentrate, and $\lambda_{0}, \lambda_{1}$, and $\lambda_{2}$ are coefficients. It means that the TFe content of sintered ores is not only related to the mixing ratio of concentrates in the mixture and the TFe content of the mixture, but also associated with the coke in the mixture.

\subsection{Modeling of alkalinity of sintered ores}

Suppose that $100 \mathrm{~kg}$ of mixture is prepared, the $\mathrm{CaO}$ and $\mathrm{SiO}_{2}$ in the mixture basically remain consistent with those in the sintered ores. Owing to the consumption of all types of refined iron-mineral powders and coke in the sintering process, the mechanism model of alkalinity of the sintered ores is shown as Formula (4).

$$
Y_{\mathrm{Alkali}}=\frac{m_{c a o} \times\left(\alpha_{0}+\alpha_{1} * x_{c}+\alpha_{2} * x_{c}^{2}\right)}{m_{s i o_{2}} \times\left(100-\sum_{i=1}^{n}\left(100 \times x_{i} \times d_{i}\right)\right)}
$$

Where $m_{\text {cao }}$ and $m_{\text {sio }_{2}}$ denote the masses of $\mathrm{CaO}$ and $\mathrm{SiO}_{2}$ in the mixture, respectively; and $\alpha_{0}, \alpha_{1}$, and $\alpha_{2}$ are coefficients.

\section{ELM based error compensation model}

As mechanism prediction models for quality of sintered ores cannot describe the strong coupling and high nonlinearity, the errors in prediction of the models are applied as the modeling objective to establish the error compensation model based on ELM. Owing to equipment parameters slowly change with time in the sintering process of iron ores and the longer the time is, the smaller the reference value of samples, the sample with longest time is assigned with the least weight.

The semi-partial correlation analysis is carried out at first to obtain factors substantially influencing the quality of sintered ores. On this basis, the error 
compensation models of the mechanism models are constructed. The input variables of the ELM based compensation models include coke content, percentage contents of $\mathrm{CaO}, \mathrm{SiO}_{2}, \mathrm{FeO}$, and each type of concentrates in the mixture, ignition temperature, movement speed of pallets, percentage content of coke, and final temperature. The output variables include compensation amounts of drum index, iron content, and alkalinity. The output of the hybrid model based on the mechanism models and the ELM based error compensation models is the sum of the output of the mechanism models and the compensation amounts of the compensation models.

Given a dataset $\left(x_{i}, y_{i}\right)$ containing $\mathrm{N}$ samples, $x_{i}=\left[x_{i 1}, x_{i 2}, \mathrm{~L} x_{i n}\right]^{T} \in R^{n}$ and $y_{i}=\left[y_{i 1}, y_{i 2}, \mathrm{~L}, y_{i m}\right]^{T} \in R^{m}(i=1,2, \mathrm{~L}, N)$. Assume that the hidden layer contains $L$ nodes and the activation function is $g(x)$, then the ELM model can be expressed as ${ }^{[6]}$

$\sum_{i=1}^{L} \beta_{i} g\left(a_{i} x_{j}+b_{i}\right)=y_{i}, j=1,2, \mathrm{~L}, N$

Where $a_{i}$ and $\beta_{i}$ represent connection weights and $b_{i}$ refers to the offset. Formula (5) can be rewritten as

$$
H \beta=Y
$$

Where

$$
\begin{aligned}
& H=\left[\begin{array}{ccc}
g\left(a_{1} \cdot x_{1}+b_{1}\right) & \mathrm{L} & g\left(a_{L} \cdot x_{1}+b_{L}\right. \\
\mathrm{M} & \mathrm{M} & \mathrm{M} \\
g\left(a_{1} \cdot x_{N}+b_{1}\right) & \mathrm{L} & g\left(a_{L} \cdot x_{N}+b_{L}\right)
\end{array}\right]_{N \times L} \\
& \beta=\left[\begin{array}{c}
\beta_{1}^{T} \\
\mathrm{M} \\
\beta_{L}^{T}
\end{array}\right]_{L \times m}, Y=\left[\begin{array}{c}
y_{1}^{T} \\
\mathrm{M} \\
y_{L}^{T}
\end{array}\right]_{N \times m}
\end{aligned}
$$

Where $H$ represents the output matrix of the hidden layer of the neural network and the output weight can be calculated by solving Formula (7).

$$
\beta=H^{+} Y
$$

Where $\mathrm{H}^{+}$is the Moore-Penrose generalized inverse matrix of the output matrix $H$ of hidden neurons.

In the training process of ELM, the samples trained for a longer time are assigned with smaller weights.

\section{Simulation verification}

The simulation results of the drum index, TFe content, and alkalinity based on the mechanism models and the hybrid model (based on the mechanism models and the ELM based compensation models) are shown in Figures 2, 3, and 4, respectively. In the figures, AV represents actual values, while $\mathrm{MM}$ and $\mathrm{HM}$ denote the simulation results based on the mechanism models and the hybrid model. Obviously, the accuracy of prediction results of the hybrid model is much higher than that when the mechanism models are used alone.

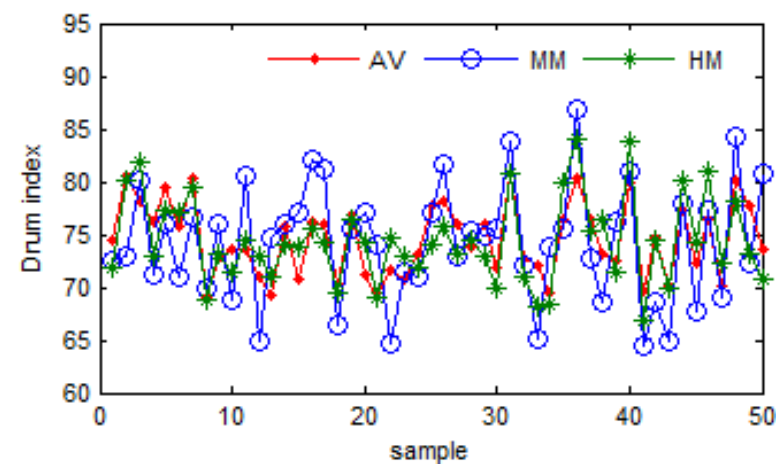

Figure 2 Simulation results of drum index

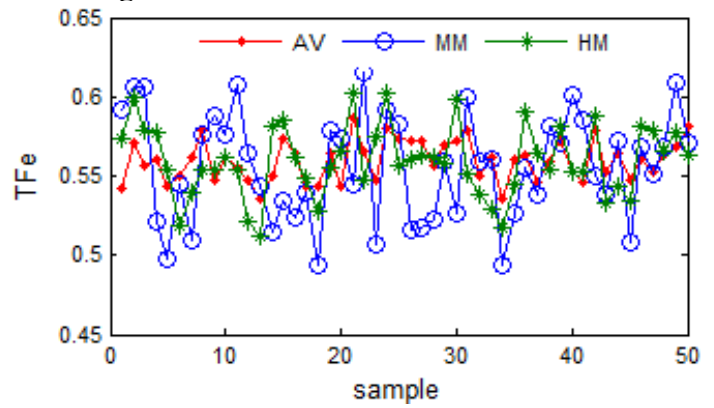

Figure 3 Simulation results of $\mathrm{TFe}$

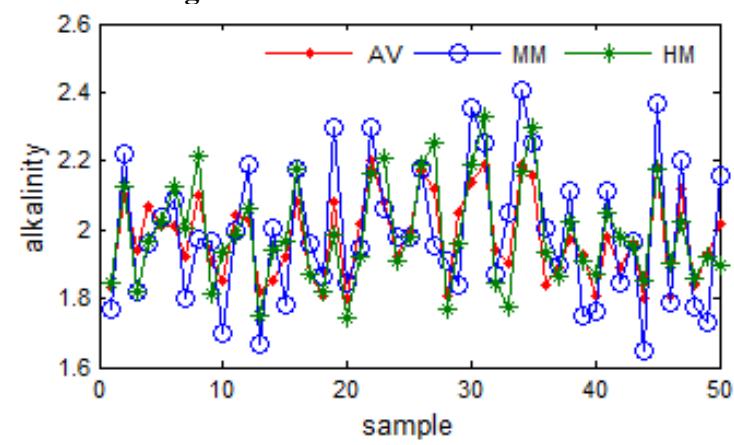

Figure 4 Simulation results of alkalinity

\section{Conclusions}

The research proposes the quality prediction model for sintered ores based on mechanism models and ELM based time-weighted error compensation. The simulation results verify that the hybrid model has much higher accuracy than that when mechanism models are used alone.

\section{Acknowledgments}

This work was supported by the Natural Science Foundation of Hunan Province (2016JJ3079), and supported by the Loudi Science and Technology Project(2017), and supported by the youth project of the Hunan Institute of Humanities and technology (2016QN18)

\section{References}


1. Liu Chengsong, Li Jing, Gao Ya Wei, Tang Haiyan. Optimization of ore matching for iron ore fines [J]. steel, 2013,48 (10): 6-11.

2. P. Lacroix, G. Dauwels, P.Dufresne, R. Godijn. High blast furnaces productivity operations with low coke rates in the European Union. LaRevue de Métallurgie-CIT, 2001, 3(1): 259-268

3. F.Patission.Study of Moister Transfer during the Strand Siniering Proeess. Metal Transacrions, 1990, 2(21B):37-47

4. Shang Xiu Qin. A new evolutionary computation method and its application in modeling and optimization of ironmaking and sintering process [D]. Hangzhou: Zhejiang University, 2010

5. numerical simulation and experimental verification of the sintering process of Zhao gape iron ore [D]. Hangzhou: Zhejiang University, 2012

6. Huang G B, Zhu Q Y, Siew C K.Extreme learning machine : theory and applications[J]. Neurocomputing, 2006, 70: 489-501. 